\title{
An Updated “Historical” Algorithm for Hirschsprung Patients Who Present Late or Severely Ill*
}

\author{
Samuel M. Alaish ${ }^{1 \#}$, Eric D. Strauch ${ }^{1}$, Steven Stylianos ${ }^{2}$ \\ ${ }^{1}$ Division of Pediatric Surgery, Department of Surgery, University of Maryland School of Medicine, Baltimore, USA; ${ }^{2}$ Division of \\ Pediatric Surgery, Department of Surgery, Cohen Children's Medical Center, North Shore-LIJ Health System, New Hyde Park, New \\ York, USA. \\ Email: *salaish@smail.umaryland.edu
}

Received May $9^{\text {th }}$, 2013; revised June $10^{\text {th }}, 2013$; accepted June $26^{\text {th }}, 2013$

Copyright (c) 2013 Samuel M. Alaish et al. This is an open access article distributed under the Creative Commons Attribution License, which permits unrestricted use, distribution, and reproduction in any medium, provided the original work is properly cited.

\begin{abstract}
Some children with previously undiagnosed Hirschprung's disease present severely ill at an advanced age with markedly dilated bowel and enterocolitis. In this manuscript, the authors propose the following treatment algorithm: 1) a diverting transverse loop colostomy, 2) a transanal Soave endorectal pull-through, and 3) colostomy closure. The authors report their experience with five patients.
\end{abstract}

Keywords: Hirschsprung’s Disease; Algorithm; Transanal Soave

\section{Introduction}

Over 50 years ago, Orvar Swenson [1] provided the first evidence that a defect of innervation was central to the disease named after Harold Hirschsprung. Subsequently, he ushered in the three-stage surgical algorithm for the treatment of these patients. The algorithm was: 1) diverting cecostomy or transverse loop colostomy, 2) Swenson pull-through procedure, and 3) colostomy closure [2]. The three-stage algorithm remained standard until the 1970s when earlier diagnosis enabled modification. First, the algorithm was shortened to two stages: 1) a leveling colostomy or ileostomy and 2) simultaneous pull-through with stomal closure. Then, in 1980, the algorithm was shortened to a single-stage in many newborns: definitive transabdominal pull-through without colostomy [3].

Laparoscopy and its applications to pediatric surgery led to the single-stage laparoscopic pull-through procedure [4]. Acknowledging that the extent of aganglionosis is in the rectosigmoid region in approximately $80 \%$ of Hirschsprung patients, Drs. De la Torre-Mondragon and Ortega-Salgado developed an exclusively transanal approach to the pull-through procedure for this group of patients and reported such in 1998 [5]. Similarly in 1999,

"Contributors: Samuel M. Alaish wrote the first draft of this paper. All authors contributed to the intellectual content and approved the final version. Samuel M. Alaish is the guarantor.

${ }^{\#}$ Corresponding author.
Langer et al. [6] reported that the pull-through procedure could be done as a single-stage using this transanal approach without the need for laparotomy or laparoscopy. Subsequently in 2003, Langer et al. [7] reported a large multicenter experience of 141 children undergoing a one-stage transanal Soave pull-through. The series demonstrated this approach to be safe, permit early feeding, cause minimal pain, facilitate early discharge and carry a low rate of complications.

Although the single-stage algorithm is now the standard of care for most Hirschsprung patients with early diagnosis, it is not applicable to all. Some infants and children present at an advanced age with markedly dilated bowel (Figure 1) or enterocolitis. On some occasions, these patients are severely malnourished or septic and require an urgent ostomy. For the remainder who are not ill, the marked dilatation of the bowel precludes a primary pull-through procedure. For this select group of patients, we advocate a new "historical" surgical management, rather than the current standard leveling colostomy, followed by an open transabdominal pull-through procedure. Our novel approach uses a smaller abdominal incision coupled with a transanal approach later for the pull-through.

\section{Methods}

Following fluid resuscitation and treatment of sepsis, a rectal biopsy is performed to make the diagnosis. During 
the time period required for this work-up, children undergo frequent rectal irrigations to evacuate stool. Once the active enterocolitis episode is resolved and the patient is clinically well (i.e. tolerating a regular diet, normal stool effluent following rectal irrigations, and afebrile with negative blood cultures (2 - 7 days in our series), a contrast enema aids in locating the transition zone (Figure 2). A few children might be too ill even for this and require urgent decompression with an ostomy. In these cases, the diagnosis can be made at the time of the ostomy. We do not advocate a contrast enema examination in the setting of acute enterocolitis due to a concern for perforation.

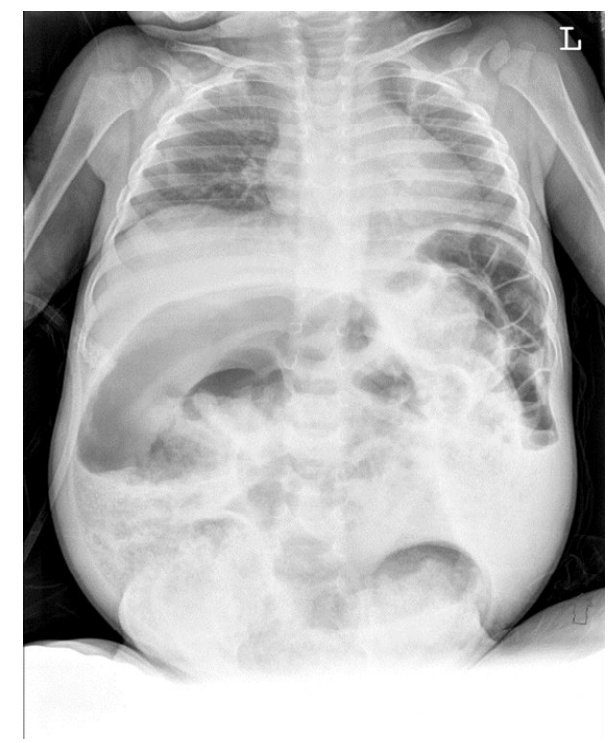

(a)

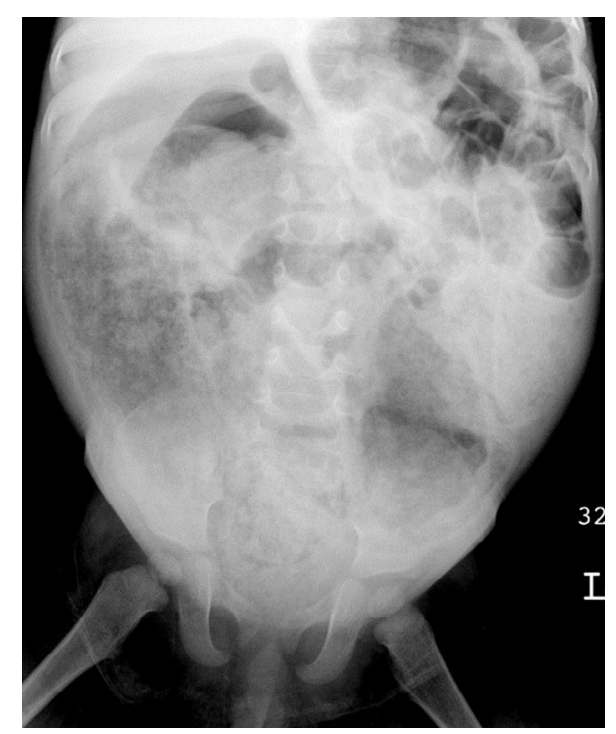

(b)

Figure 1. Abdominal radiographs of 19 month-old child upon initial presentation. Note the marked distension of the sigmoid colon.

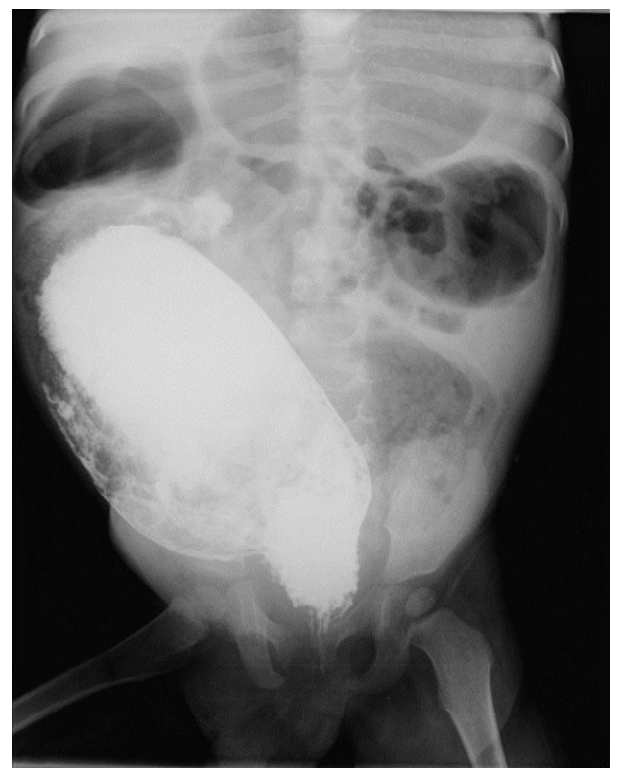

(a)

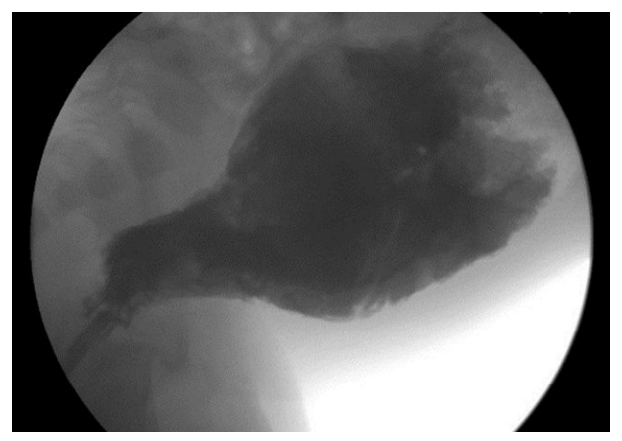

(b)

Figure 2. Images taken during contrast enema performed on the same patient. The transition zone is clearly seen.

For children who present late or severely ill and are found to have a transition zone in the left colon (approximately $80 \%$ of patients), we propose the algorithm depicted in Figure 3.

Our approach creates a more proximal colostomy using bowel that is less dilated than that found at the transition zone; this enables us to use a smaller abdominal incision for the stoma. A transverse loop colostomy can be performed expeditiously through a small right upper abdominal incision. A biopsy is sent for frozen section to confirm the presence of normal ganglion cells and the absence of hypertrophied nerve fibers. The stoma is then brought out through the incision. When done properly, this transverse colostomy will not jeopardize the subsequent pull-through procedure. Following an adequate period of time ( 3 - 6 months) for the caliber of the bowel to shrink sufficient for a pull-through procedure, a contrast enema is performed to confirm just that.

With a transition zone identified in the left colon on the initial contrast enema, the vast majority of patients 


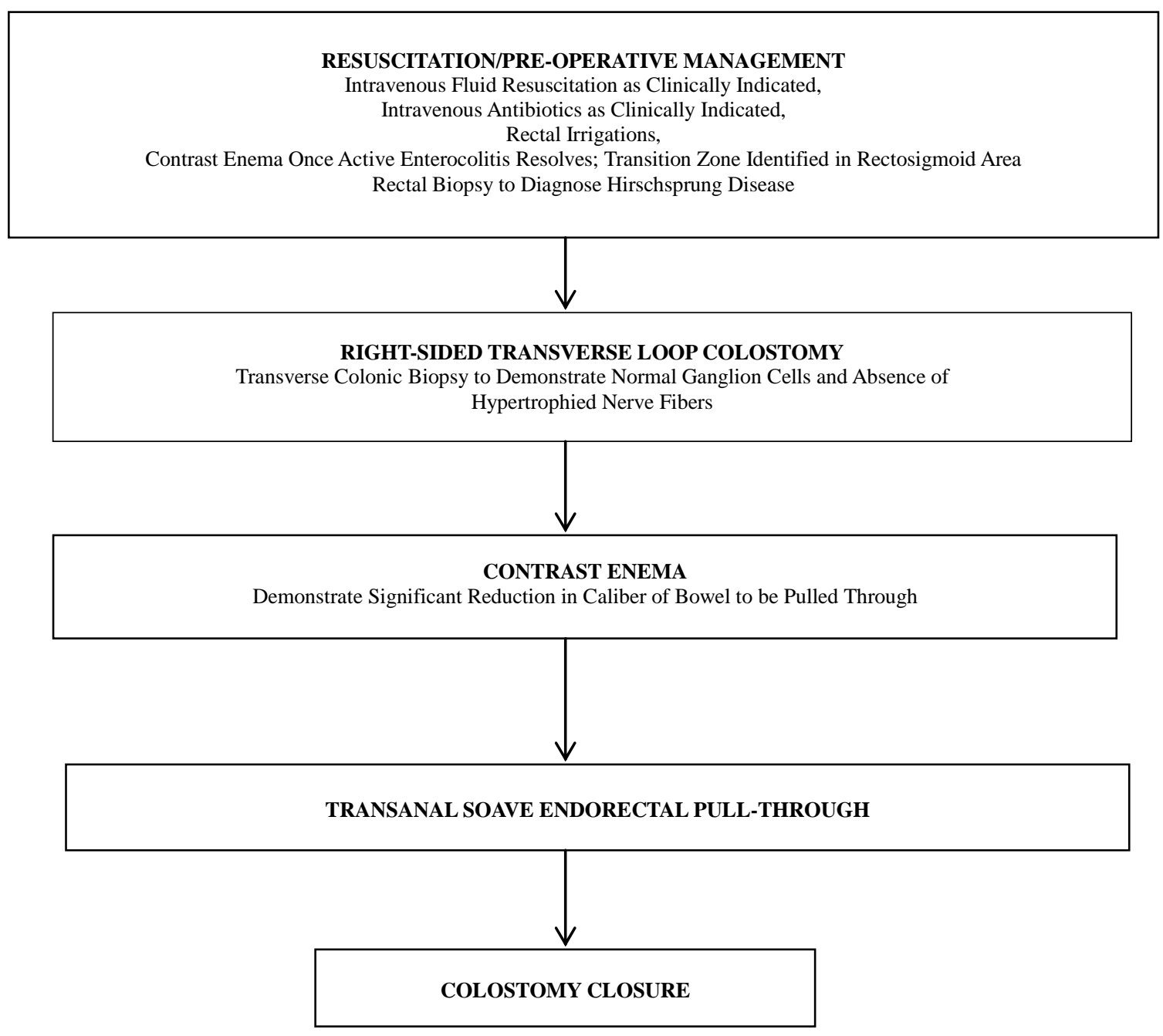

Figure 3. Proposed algorithm for Hirschsprung patients who present late or severely ill.

can undergo a transanal Soave pull-through without anterior abdominal wall access. Nonetheless, the surgeon should be prepared to use laparoscopic assistance or a counter-incision in the anterior abdominal wall, should additional mobilization be needed, or should the transition zone be more proximal than that read from the barium enema. The third and final operation in the algorithm is a loop colostomy takedown performed 4 to 6 weeks following the second operation.

\section{Results}

We have applied this algorithm thus far to 5 patients. The ages at initial presentation were 11 days, 9 months, 15 months, 17 months, and 19 months. All five patients had poor feeding and weight gain in addition to their "constipation". None of the 5 patients had other anomalies. All five patients had clinical grade II enterocolitis and were managed preoperatively with rectal irrigations and broad-spectrum antibiotic therapy. Standard rectal irriga- tions were begun immediately and continued preoperatively as follows: normal saline rectal washouts $(10 \mathrm{ml} /$ kg volume for each washout) were administered through a large caliber (18 - 20 Fr) rectal tube three times a day [8]. The catheter for the washouts was placed above the aganglionic portion and used to decompress the bowel as traditionally recommended. All patients demonstrated a rectosigmoid transition zone on contrast enema. Unfortunately, rectal irrigations were inadequate in our 11 day-old patient. He continued to feed poorly, and his nutritional status worsened. Rather than proceed towards a primary pull-through, which would be standard for someone of this age who was adequately nourished, we elected to perform a diverting transverse loop colostomy in the same manner as our older patients. All patients thrived following colostomy. The algorithm was completed over 5 to 9 months. The mean follow-up is 9 years (range 23 months-10 years). There have been no deaths and no complications (anastomotic leaks, postoperative enterocolitis, incontinence, bowel obstruction, stomal 
stenosis or stomal prolapse). All patients enjoy normal spontaneous bowel movements without soiling or the need for enemas or laxatives.

\section{Discussion}

Children with Hirschsprung's disease who present outside the newborn period have marked distension of the bowel proximal to the transition zone. The vast majority of these patients have a transition zone in the rectosigmoid region [9]. They can be malnourished and often present with enterocolitis. The current management for these patients in most centers worldwide would be the creation of a leveling loop colostomy. A loop colostomy is done rather than an end colostomy with closure of the distal bowel, because the latter approach results in a functional closed loop obstruction which could lead to perforation with significant morbidity and possible mortality. Unfortunately, the marked dilation of the bowel makes a leveling colostomy problematic due to the common complications of stomal prolapse and peristomal hernias [8]. These complications develop as the child recovers and the bowel shrinks. In this manuscript, we propose a novel algorithm for this select group of patients combining lessons learned from the past with some of the most recent technical advances in pediatric surgery.

Contrary to a recent article advocating primary transanal endorectal pull-through for adolescents and adults who present late [10], we believe children who present outside the newborn period are not candidates for a primary pull-through because of the marked distension of their bowel. In the study by Ammar et al. [10], the adolescents and adults may constitute a different patient population with a different disease severity. Following only 2 days of rectal irrigations, a primary pull-through was performed using the transanal approach. The authors state in their article that bowel was resected until the caliber was nearly normal. This implies that the aganglionic segment was quite short with limited proximal dilatation. Nevertheless, the high rate of incomplete incontinence in the form of soiling (26.6\%) in their series [10] is concerning and quite different from our experience. Excessive retraction on the anal sphincters to accommodate dilated bowel can lead to incontinence, and we avoid that.

Rectal irrigations for a period of several months to reduce the caliber of the bowel are impractical for these active children. An ostomy is indicated. For the three toddlers in our series, the colostomy provided reliable stool evacuation and bowel decompression without the need for rectal irrigations, which can be traumatic for both the patient and the provider in these older, stronger children. This benefit was also enjoyed by the 9 month- old infant, for he was walking prior to the time required for the bowel to shrink sufficiently to accommodate a pull-through procedure. Our 11 day-old patient was somewhat of an outlier. The majority of infants this young can be managed with rectal irrigations followed by a primary pull-through procedure. This was exactly our initial plan; however, the rectal irrigations failed to improve his clinical course. He continued to feed poorly in the hospital, and his poor nutritional state on presentation only worsened while in the hospital. Over time, it became clear that a colostomy was the best option for him. Postoperatively, he quickly thrived.

Following diversion the dilated ganglionated bowel will shrink over time and function well. There is no need for resection. Complications with leveling colostomies occur in these children due to the marked dilatation of the bowel. Therefore, we advocate a more proximal transverse right colostomy in this select group of patients. This can be performed expeditiously through a small incision. We must emphasize that a normal biopsy at this level is mandatory prior to the creation of a stoma. Should the biopsy be abnormal, then our algorithm would be aborted in favor of the standard leveling ostomy, which would result in an even more proximal stoma located in either the ascending colon or the small intestine.

The second operation in our algorithm is performed a few months later. This time period enables the child to "catch up" from a nutritional standpoint and also allow the bowel to shrink to a size suitable for a pull-through operation. Pull-through operations in these patients generally involve difficult dissections due to the patient's advanced age and previous bouts of enterocolitis. The diverting colostomy is maintained to reduce the length of operative time required for the second operation and reduce potential complications. We are concerned that coloanal anastomotic dehiscence without a protective stoma may lead to excessive scarring and decreased continence; however, we are aware that colostomies in infants and children are associated with significant complications including prolapse, stenosis, retraction, dysfunction, skin excoriation and parastomal hernia. In addition, colostomy closure carries its own morbidity [11]. In our small series, we have not encountered any morbidity related to the stoma; however, if significant morbidity surfaces in future patients, shortening the algorithm to two operations may be warranted. The colostomy closure would then be performed at the time of the pull-through procedure. We acknowledge that our series is limited by its small size; however, this is a select group of patients which are not typically encountered at any one institution in large numbers. Despite this shortcoming, we believe our novel approach offers a safe and straightforward option for these select patients which results in smaller ab- 
dominal incisions and potentially less stomal complications, improved cosmesis, less postoperative pain and faster recovery.

\section{REFERENCES}

[1] O. Swenson, "My Early Experience with Hirschsprung's Disease," Journal of Pediatric Surgery, Vol. 24, No. 8, 1989, pp. 839-845. doi:10.1016/S0022-3468(89)80549-4

[2] O. Swenson and A. H. Bill, "Resection of Rectum and Rectosigmoid with Preservation of the Sphincter for Benign Spastic Lesions Producing Megacolon," Surgery, Vol. 24, No. 2, 1948, pp. 212-220.

[3] H. B. So, D. L. Schwartz, J. M. Becker, F. Daum and K. M. Schneider, “Endorectal 'Pull-Through' without Preliminary Colostomy in Neonates with Hirschsprung's Disease,” Journal of Pediatric Surgery, Vol. 15, No. 4, 1980, pp. 470-471. doi:10.1016/S0022-3468(80)80755-X

[4] K. Georgeson, M. Fuenfer and W. Hardin, "Primary Laparoscopic Pull-Through for Hirschsprung's Disease in Infants and Children,” Journal of Pediatric Surgery, Vol. 30, No. 7, 1995, pp. 1017-1022. doi:10.1016/0022-3468(95)90333-X

[5] L. De la Torre-Mondragon and J. A. Ortega-Salgado, "Transanal Endorectal Pull-Through for Hirschsprung's Disease,” Journal of Pediatric Surgery, Vol. 33, No. 8, 1998, pp. 1283-1286. doi:10.1016/S0022-3468(98)90169-5

[6] J. C. Langer, R. K. Minkes, M. V. Mazziotti, M. A. Skin- ner and A. L. Winthrop, "Transanal One-Stage Soave Procedure for Infants with Hirschsprung's Disease,” Journal of Pediatric Surgery, Vol. 34, No. 1, 1999, pp. 148-152. doi:10.1016/S0022-3468(99)90246-4

[7] J. C. Langer, A. C. Durrant, L. de la Torre, D. H. Teitelbaum, R. K. Minkes, M. G. Caty, B. E. Wildhaber, S. J. Ortega, S. Hirose and C. T. Albanese, "One-Stage Transanal Soave Pullthrough for Hirschsprung Disease: A Multicenter Experience with 141 Children," Annals of Surgery, Vol. 238, No. 4, 2003, pp. 569-583.

[8] D. H. Teitelbaum, M. L. Walkun, K. E. Georgeson and J. C. Langer, "Hirschsprung's Disease,” In: M. M. Ziegler, R. G. Azizkhan and T. R. Weber, Eds., Operative Pediatric Surgery, McGraw-Hill Companies, New York, 2003, pp. 617-645.

[9] D. H. Teitelbaum and A. G. Coran, "Hirschsprung’s Disease and Related Neuromuscular Disorders of the Intestine,” In: J. L. Grosfeld, J. A. O’Neill Jr., E. W. Fonkalsrud and A. G. Coran, Eds., Pediatric Surgery, Mosby, Inc., Philadelphia, 2006, pp. 1514-1559.

[10] S. A. Ammar, I. A. Ibrahim, “One-Stage Transanal Endorectal Pull-Through for Treatment of Hirschsprung's Disease in Adolescents and Adults,” Journal of Gastrointestinal Surgery, Vol. 15, No. 12, 2011, pp. 2246-2250. doi:10.1007/s11605-011-1662-z

[11] S. Nour, J. Beck, M. D. Stringer, "Colostomy Complications in Infants and Children," Annals of The Royal College of Surgeons of England, Vol. 78, No. 6, 1996, pp. 526-530. 